\title{
THE PRESENT STATUS OF CHEMOTHERAPEUTIC AND ANTIBIOTIC DRUGS
}

There is no more important or more rapidly developing subject than that of chemotherapy. A bewilderingly increasing number of drugs are on the market. Each is stated to be effective in a great many conditions. Do these new drugs fulfil the claims made for them? Are their advantages as great clinically as in the laboratory? Can one drug be relied on in a particular condition or is a combination of drugs more effective? How do the new drugs compare with their predecessors in the field, in both action and in reactions?

The Postgraduate Medical Journal is pleased and proud to announce the following series of articles on the subject of the present position of the Chemotherapeutic and Antibiotic Drugs. Written by acknowledged experts in the various fields, they have been planned specifically to give the clinician the most up-todate, practical information on each subject. In so progressive a field it is more than ever important to keep up to date. The Editors are confident that this series will prove of the greatest interest and value The first of the series, on the subject of Chemotherapy and the Venereal Diseases, appears in this presen issue; the others will appear in successive numbers.

Chemotherapy and the Venereal Diseases

R. R. Willcox M.D.

The Chemotherapy of Tropical Infections of the Bowel

The Chemotherapy of Non-Tuberculous Diseases of the Chest

The Chemotherapy of Pulmonary Tuberculosis

The Chemotherapy of Non-Pulmonary Tuberculosis

Recent Advances in Malaria

Prof. F. Murgatroyd M.D., F.R.C.P.

The Chemotherapy of Tuberculous Infections of the Urinary Tract

A. R. D. Adams M.D., F.R.C.P.

\section{Horace Foules} M.D., F.R.C.P.

7. G. Scadding M.D., F.R.C.P.

$$
\begin{aligned}
& \text { P. Buxton } \\
& \text { M.R.C.P. }
\end{aligned}
$$

Arthur Facobs F.R.F.P.S.

The Chemotherapy of Non-Tuberculous Infections of the Urinary Tract

F. R. Kilpatrick M.S., F.R.C.S.

The Most Recent Antibiotic Agents

G. Brownlee Ph.D., D.Sc. 